kaum der Hoffnung hin, dass ich den Leser schon jetzt zu meinen Ansichten bekehrt habe. Nur soviel hoffe ich bewiesen zu haben, dass die bisher gangbare Lehre von der Natur der Innervation der Gefässe den Thatsachen nicht gerecht wird. Wenn Jemand zur Erklärung der von mir gefundenen Thatsachen eine bessere Hypothese findet, als die meine ist, so würde ich diese gern aufgeben.

Im hiesigen Laboratorium werden gegenwärtig neue Versuche vorbereitet, um einige Punkte der in dieser Abhandlung berührten Frage weiter aufzuklären.

\title{
Ueber die Abhängigkeit der Farbenempfindung von der Zeit.
}

Von

\section{Dr. A. Kunkel,}

Assistenten am physiologischen Institut zu Würzburg.

Hierzu Tafel Va.

Der continuirlichen Folge verschiedenor Länge der Aetherwellen im Spectrum entspricht nicht eine gleiche continuirliche Folge verschiedener Empfindungen. Es ist vielmehr aine specifische Eigenscbaft der das Licht percipirenden nervösen Endapparate durch grössere Theile des Spectrum, deren einzelne Abschnitte nichts mit einander gemein haben als eine geringere Differenz in der Wellenlänge, in solcher Weise erregt zu werden, dass identische Empfindungen zu Stande kommen, und so unterscheidet das gemeine Bewusstsein im Spectrum gewisse Principalempfindungen - Roth, Gelb, Grün, Blau - zwischen denen wohl continuirliche Uebergänge auch unserer Empfindung Statt haben, welch letztere Empfindungen indess wir nur als Resultirende der beiden angrenzenden Principalempfindungen dem gewöhnlichen Sprachgebrauche gemäss auffassen.

Eine jede Untersuchung des Farbensinnes wird natürlich zunächst diese Principalempfindungen zum Objecte wählen. Ich habe in der Untersuchung, deren Resultate ich hier mittheilen werde, die Theile des Spectrum, die den Empfindungen roth, grün und blau angehören, als Beobachtungsgegenstand gebraucht. Gelb habe ich 
nicht aus Voreingenommensein für eine Theorie, sondern nur desshalb ausgeschlossen, weil es trotz starker Dispersion nicht möglich war, ein hinreichend grosses Gesichtsfeld mit reinem, homogenem Gelb zu erfüllen.

Ich hatte mir vorgenommen, die Abhängigkeit der Farbenempfindung von der Zeit zu untersuchen, im Einzelnen die Frage gestellt, welche Zeit die verschiedenen Theile des Spectrum brauchen, um eine bestimmte Netzhautparthie in das Maximum von Erregung $\mathrm{zu}$ versetzen. Es handelte sich hierbei um Helligkeitsbestimmungen. Ich bemerke hierzu gleich von vorne herein, dass die drei Grössen, die man an einer Farbenempfindung unterscheidet, Helligkeit, Sättigung und Farbenton, in einem ganz bestimmten Abhängigkeitsverhältniss zu einander stehen: am einfachsten werden die hier auftretenden Erscheinungen durch die Annahme erklärt, Farbenton und Sättigung als abhängig von der Helligkeit - innerhalb gewisser Grenzen zu betrachten. Es ist natürlich nothwendig, bei ad hoc angestellten Versuchen immer nur die eine dieser Beziehungen als die zunächst in Frage stehende messend $z \mathfrak{u}$ verfolgen.

Es war schon von Platea ${ }^{1}$ ) gelegentlich die Bemerkung gemacht worden, dass es "zur vollen Bildung eines Lichteindrucks" einer gewissen endlichen Zeit bedürfe. Den gleichen Satz hat A. Fick ${ }^{2}$ ) durch theoretische Ueberlegungen aus dem Talbot'schen Satze gefolgert und unter Voraussetzung der Wahrheit dieses Satzes eine ganz bestimmte Abhängigkeit zwischen dem "Anklingen " und Abklingen einer Erregung festgestellt. Auch hat Fick am gleichen Orte den obigen Satz experimentell bewiesen. Ungefähr gleichzeitig hat E. Br ü cke ${ }^{3}$ ) denselben Gedanken in einem bestimmten Einzelfalle durch das Experiment ausgeführt und die Zeit, die ein bestimmter Lichtreiz zur Hervorbringung maximaler Erregung gebraucht, zu 0,18 Sec. gefunden. Aubert ${ }^{4}$ ) gibt bei Besprechung von Fick's Resultaten Versuche an, die Zeit für das Maximum der Erregung

1) Ueber einige Eigenschaften der vom Lichte auf das Gesichtsorgan hervorgebrachten Eindrücke: Poggendorf Annal. Jahrg. 1830 Bd. XX. pag. 307.

2) Ueber den zeitlichen Verlauf der Erregung in der Netzhaut: du Bo is und Reichert's Archiv 1863 pag. 739 ff.

3) Ueber den Nutzeffect intermittirender Netzhautreizung: Wiener Acad, Ber. Jahrg. 1864. pap. 132 und 133.

4) Physiologie der Netzhaut pag. 353. 
zu bestimmen und findet Zahlen, die mit den durch vollkommenere Methoden gewonnenen recht gut stimmen. In einer grösseren Arbeit hat dann S. Exner ${ }^{1}$ ) mittelst eines von Helmholtz construirten Apparates diese Zeit unter verschiedenen Bedingungen durch den Versuch ermittelt. Beobachtungsobject waren Exner mit weissem Papier überzogene Scheiben, die er mit Gasflammen beleuchtete.

Zur Bestimmung der Zeit, die nothwendig ist, damit verschiedenes, homogenes Licht die Netzhaut in das Maximum von Erregung versetzt, benutzte ich den oben erwähnten Apparat, mittels dessen es möglich ist, einen Lichtreiz durch beliebig lange, genau messbare Zeit auf das Auge einwirken zu lassen. Es besteht dieser Apparat (cf. Die schematische Zeichnung Fig. 1 a) wesentlich aus zwei Scheiben $A$ und $B$, die fest mit Axen verbunden sind, welche verlängert in eine einzige Gerade zusammenfallen und die, wie direct ersichtlich, in gleichem Sinne aber mit verschiedener Geschwindigkeit rotiren. Es ist die Uebertragung der rotirenden Bewegung von $B$ auf $A$ vermittelst der auf der Axe a befestigten Zahnräder $b$ und $c$ so eingerichtet, dass B eine gerade zwölfmal grössere Winkelgeschwindigkeit besitzt als A. B ist mit etwas kleinerem Radius beschrieben als A. Eine auf der gleichen Axe mit B beweglich angebrachte, gleich grosse Metallscheibe (die eigentlich in der Zeichnung allein sichtbar ist), kann durch eine Schraubenmutter $m$ gegen $B$ fest angedrückt werden. Durch diese Vorrichtung ist es möglich, Papiersectoren von grösserem Halbmesser als B zwischen dieses und die bewegliche Metallplatte einzuklemmen. Es kann so ein leuchtendes Object, das hinter der rotirenden Scheibe B und zwar in der Höhe $\alpha \beta$ steht, durch Zurückschieben bezw. Aufsteken von Papiersectoren beliebig lange Zeit nach der Scheibe A hin Licht aussenden oder verdunkelt werden. Die Scheibe A trägt an ihrer Peripherie einen Ausschnitt $C$ von genau der Breite $\alpha \beta$ - der Breite dẹr Papiersektoren - der nur 1/12 der Peripherie einnimmt. Hinter A (nach links in der Zeichnung vom Beschauer aus) ist ein durch eine Schraube verschieden weit stellbarer Spalt angebracht, durch welchen man eine beliebige Menge des auf A fallenden Lichtes benutzen kann. Dicht hinter dem Spalt steht der Beobachter. Da die Beobachtung durch ein Fernrohr geschieht, so kommt also unmittelbar hinter diesen Spalt bei directer

1) Ueber die zu einer Gesichtswahrnehmung nöthige Zeit. Wien. Sitz. Ber. Jahrg. 1868. pag, $601 \mathrm{ff}$. 
Beobachtung das Objectiv des Fermrohres. Bei Wegnahme aller Papiersektoren von $\mathrm{B}$ würde dem hinter $\mathrm{A}$ beobachtenden Auge das leuchtende Object genau so lange erscheinen, als B zu einer Umdrehung gebraucht. Das leuchtende Object wird natürlich dem wie oben beschrieben aufgestellten Auge immer nur sichtbar sein, wenn $\mathrm{C}$ den höchsten Stand an der Peripherie von A einnimmt, also immer nach je 12 Umdrehungen von B. Es ist dies der Zweck der Scheibe A, das leuchtende Object immer nur in/ grösseren Intervallen dem Auge wieder darzubieten, damit nicht durch zu rasch sich folgendes Erscheinen die Genauigkeit der Einzelbeobachtung beeinträchtigt werde. Die Scheibe B trägt eine Kreistheilung, mittels welcher man direkt den Stand der eingeschobenen Sektoren beobachten, und daraus mit Hilfe der bekannten Winkelgeschwindigkeit der Scheibe die Dauer des Vorübergangs der an B freigelassenen Oeffnung berechnen kann. Auf der Axe der Scheibe B sitzt eine Scheibe, die am Rande eine Nute trägt, welche durch einen Schnurlauf mit einem Motor verbunden ist. Natürlich muss die Rotation möglichst constant und willkürlich zu ändern sein.

Die Methode von Exner, die Zeit zu bestimmen, nach welcher eine maximale Erregung $\mathrm{zu}$ Staude gekommen, ist die folgende:

Es sei a bcd in Fig 1 in graphischer Darstellung der Verlauf der Erregung einer bestimmten Netzhautstelle, wobei die Abscissen die Zeit und die Ordinaten die den einzelnen Zeittheilchen entsprechenden Erregungswerthe bedeuten. Es sei dabei der ganze Verlauf einer Erregung in der Form der Curve (Anklingen, Maximum, Abfallen durch Ermüdung) einstweilen vorausgesetzt. Wenn nun eine bestimmte Netzhautstelle durch das leuchtende Object, das aus einem halb weissen, halb schwarzen Schirm besteht, in den Beginn der Erregung versetzt wird, welche nach der Curve a bcd verlaufen würde und dann dem Auge nach sehr kurzer Dauer, welche durch das Stückchen a $\alpha$ vorgestellt ist, ein leuchtendes Object dargeboten wird, welches nun das ganze Gesichtsfeld erfüllt, so wird, falls die Beleuchtungsintensität dieses zweiten Objectes nur genau die gleiche ist, die Erregung der zuerst gereizten Netzhautstelle nun wirklich nach der Curve abcd ablaufen, zugleich aber wird die zunächst gelegene Netzhautstelle, auf welche während der Zeit a $\alpha$ das Bild des schwarzen Schirmtheiles gefallen war, nun in Erregung versetzt werden, welche ihrerseits nach der Curve $\alpha \beta \gamma \delta$ abläuft. Beide Curven schneiden sich in $f, d, h$. es wird an diesem 
Punkte kein Helligkeitsunterschied zwischen den beiden Hälften des Gesichtfeldes mehr existiren. Schneidet man also an dem Punkte $f$, d, h nach der Zeit ag die Reizung ab, in dem Momente also, wenn die beiden Hälften des Gesichtsfeldes gleich hell erscheinen, so hat die Erregung, die a b c d entspricht, das Maximum bereits um etwas überschritten, $\alpha \beta \gamma \delta$ noch nicht erreicht. An jeder andern Stelle abgeschnitten, muss sich ein Helligkeitsunterschied in dem einen oder andern Sinne ergeben. -

Für den Anfangstheil der Curve bis $\beta$ b ist dieser Helligkeitsunterschied sehr deutlich und ist ein entschiedener Beweis der Richtigkeit des dieser ganzen Betrachtung zu Grunde liegenden Satzes. Dagegen verlaufen die absteigenden Theile beider Curven so nahe bei einander, dass ich wenigstens einen Helligkeitsunterschied zwischen den beiden Hälften des Gesichtsfeldes nicht erkennen konnte. In Betreff der Anordnung des Versuches ist bei Exner selbst nachzusehen.

Ich wollte zunächst als Vorversuch genau nach Exner's Vorschrift einige Versuchsreihen mit weissem Lichte anstellen. Es gelang mir indess nicht hierbei zu einem Resultate zu gelangen und so Exner's Methode zu bestätigen ${ }^{1}$ ).

1) Ich bemerke ausdrücklich, seine Methode zu bestätigen, denn seine Resultate kann ich, soweit ich sie prüfte, bestätigen. -

Was nämlich die Methode anlangt, so gelang es mir niemals, beim Abschneiden der beiden Erregungscurven an Punkten, die sicherlich jenseits des Maximum gelegen waren, die Hälfte des Gesichtsfeldes, welehe nur kürzere Zeit sichtbar gewesen war, auch nur gleich hell, geschweige denn heller zu sehen, als die dem Auge durch längere Zeit dargebotene. Allerdings behauptet auch Exner nicht das letztere gesehen zu haben. Er beschreibt die Erscheinung so, dass ihm beim Abschneiden der Reizungen an einem Punkte, der jenseits des Durchschnittes beider Curven gelegen war, der zuerst gesehene weisse Halbkreis im negativen Nachbilde erschien, das er wals hellen Streifen der durch Contrast besonders hervortretenden Grenze zwischen dem schwarzen und weissen Halbkreise beobachtete. Warum Exner diese Erscheinung in dem eben erwähnten Sinne auffasst und warum er sie gerade negatives Nachbild nennt, ist mir nicht ganz klar. Es kommt natürlich bei derlei Versuchen vor Allem darauf an, gerade den letzten Moment vor Abschneiden der ganzen Lichterscheinung im Gedächtnisse zu fixiren. Ich glaube, dass ich in diesem. Vortheile durch die vielen Versuche, die ich nach einer anderen, gleich zu besprechenden Methode angestellt habe, zu einiger Uebung und Sicherheit des Urtheils gekommen bin. Ich habe mich wegen 
Die Methode, nach der ich verfuhr, ist folgende:

Es sei a s z x y (Fig. 3) der Verlauf einer Erregungscurve, hervorgebracht durch eine bestimmte Helligkeit, wobei wieder, wie direct ersichtlich, die Abscissen die Zeit, die Ordinaten die jeweiligen Erregungswerthe darstellen.

Nun ist es Eigenthümlichkeit des Auges, dass die Erregungscurve einer zweiten, viel grösseren Helligkeit viel steiler ansteigt, als die der geringeren Helligkeit ${ }^{1}$ ). Nach den Vorstellungen, die man sich von dem Vorgange der Erregung macht, war dies auch zu erwarten. Wenn ich nun, nachdem die Erregung einer gewissen Netzhautstelle durch eine bestimmte, geringere Helligkeit während einiger Zeit schon gedauert hat, auf eine benachbarte Netzhautstelle eine zweite, viel grössere Helligkeit einwirken lasse, so werde ich leicht den Zeitpunkt suchen können, wann die beiden Netzhautstellen ungefähr in gleichem Grade der Erregung sich befinden. So würde in Fig. 3 eine Helligkeit, die eine Netzhautstelle in nach der Curve b o s u w ansteigende Erregung versetzt, schon nach der Zeit b c die gleiche Erregung hervorbringen, welche eine geringere Helligkeit, die eine benachbarte Netzhautparthie reizt und welche die Erregungscurve ats zx y v liefert, auf dieser erst nach der Zeit a c setzt. Ein gleichzeitiges Abschneiden der Erregung beider Netzhautstellen nach Zeiten a e oder a d würde Helligkeitsunterschiede in verschiedenem Sinne ergeben, wie direct aus der Zeichnung ersichtlich. Die Zeit b c nun, die nothwendig ist, damit die grössere Helligkeit eine Netzhautstelle in den gleich grossen Erregungszustand

meines Misserfolges auch brieflich mit Hrn. Exner über die Methode und die Details der Ausführung besprochen, ohne dass indess seine höchst dankenswerthe Antwort mir hinreichende Aufklärung gegeben hätte. lch kann mir den negativen Ausfall meiner nach Exner's Methode angestellten Versuche nicht erklären, da ich einmal genau nach seiner Methode verfuhr, in der Beurtheilung des Gesehenen im späteren Verlaufe meiner Untersuchungen mir hinreichende Uebung verschafft $\mathrm{zu}$ haben glaube, da ferner die Ueberlegung, die Exner's Methode zu Grunde liegt, a priori beurtheilt, durchaus richtig ist und die von mir nach einer andern Methode erhaltenen Resultate mit denen von Exner der Hauptsache nach stimmen.

1) Es ist ja a priori auch denkbar, dass die Erregungscurven zweier angrenzenden Netzhautstellen, auf die verschiedene Lichtintensitäten wirken, aussehen wie Fig. 2, so dass nach $\mathrm{mn}$, mo noch kein Helligkeitsunterschied bemerkbar wäre, sondern erst nach Zeiten m q, mp. 
versetzt, in welchen die geringere Helligkeit eine andere Netzhautstelle erst nach der Zeit a c versetzt, wird verschieden sein, je nachdem die der geringeren Helligkeit entsprechende Erregung schon einen grösseren oder kleineren Werth erreicht oder bereits ihr Maximum überschritten hat und nun (wegen der Ermüdung) den Verlauf $\mathrm{vc}^{\prime} \mathrm{d}^{\prime}$ nimmt. Es wird also die Zeit $\mathrm{bc}<\mathrm{fg}<\mathrm{kl}<\mathrm{il}$ sein, während die Zeiten il und $m \mathrm{n}$ ungefähr gleich und dann wieder $a^{\prime} b^{\prime}<m n$ sein werden. Es wird also ein gewisses Stück der Erregungscurve, d. i. einen bestimmten Zeitabschnitt im Verlaufe einer Erregung geben, während dessen die Zeiten, die eine zweite, sehr grosse Helligkeit braucht, um die gleiche Erregung hervorzubringen, ungefähr gleich sind und einen Maximalwerth besitzen, indem die nach rechts und links von dem Stücke y v gelegenen Curventheile Erregungswerthe darstellen, für die es nur kürzerer Zeiten bedarf, um durch eine zweite, beträchtliche Helligkeit hervorgebracht zu werden.

Dies ist der Gedanke, welcher der von mir angewendeten Methode $\mathrm{zu}$ Grunde liegt.

Die Ausführung geschah in folgender Weise: Die an die Scheibe $B$ (Fig. 1a) angesteckten Papiersectoren $d$ und 1 sind von verschiedener Grösse. Der Halbmesser der grösseren Sorte ist dem der Scheibe A gleich, der der kleineren ist so gewählt, dass die Breite des Ringes $\alpha \beta$ genau das Doppelte von der des Ringes $\alpha \gamma$ beträgt. Diese Papiersectoren verdecken, wie oben angegeben, die dicht hinter ihnen (nach rechts vom Beschauer) stehende Lichtquelle. Als Lichtquelle benutze ich das Bild eines Spaltes, welches genau in die Ebene der Papiersectoren und zwar an den höchsten Punkt der Peripherie von $B$ fällt. Dieser Spalt ist in eine obere und untere Hälfte getheilt, indem die bei Spalten gewöhnlich einfache bewegliche Platte durch eine horizontale Trennungslinie halbirt ist: jede Hälfte der Platte dieses Spaltes kann gesondert durch eine Mikrometerschraube bewegt werden. Diese Mikrometerschrauben gestatten durch Kreistheilungen beliebige und bekannte Aenderung der Weite der Spalthälften. Durch entsprechende Beleuchtung des Spaltes erhält man von demselben ein Bild, dessen obere und untere Hälfte durch verschieden weites Oeffnen der beiden Spalthälften verschiedene Breite besitzen. Dieses Spaltbild fällt bei zweckmässiger Aufstellung so auf die Papiersectoren, dass der untere und zwar breitere (lichtstärkere) Theil genau durch die kleineren $\mathrm{Pa}$ - 
piersectoren verdeckt wird, während der obere, schmälere (lichtschwächere) Theil genau auf die grösseren Papiersectoren fällt. Beim Stande der Papiersectoren, wie er in Fig. 1 a dargestellt ist, würde also die schmälere Hälfte des Spaltbildes während der Dauer des Vorübergangs von $\alpha \vartheta$, die breitere während der Daner $\lambda \mu$ sichtbar sein. Dieses Spaltbild wird bei passender Anordnung ein Spectrum erzeugen, dessen obere und untere Hälfte verschieden hell und zwar von bekanntem Helligkeitsverhältniss sind. Durch Verschieben der beiden Sectorenarten kann im Allgemeinen jede der beiden Bildhälften beliebig lange sichtbar gemacht werlen, mit der Einschränkung, dass die untere nur eben so lange oder durch kürzere Zeit als die obere sichtbar ist. Es wird, wie wohl direct ersichtlich, zur $\Lambda$ nstellung des oben methodisch beschriebenen Versuches die Anordnung der Papiersectoren für gewöhnlich eine solche sein, dass die obere, lichtschwächere Hälfte des Spaltbildes durch längere Zeit aufgedeckt ist, als die untere lichtstärkere Hälfte und zwar wird der Zeit nach die Aufdeckung der beiden Hälften des Spaltbildes so erfolgen, dass immer die untere, lichtstärkere Hälfte erst dann aufgedeckt wird, wenn die obere, lichtschwächere schon durch einige Zeit aufgedeckt war. Beide Hälften des Bildes werden gleichzeitig durch einen vorhergeschobenen Sector von grösserem Halbmesser wieder zugedeckt. Als die am leichtesten immer möglichst constant zu erhaltende Lichtquelle benutzte ich eine Petroleumlampe (Flachbrenner). Zur Erzeugung des Spectrums dienten zwei Prismen, ein Flintglasprisma von hohem Dispersionsvermögen und ein Schwefelkohlenstoffprisma. In dem hinter diesem aufgestellten Fernrohre erschien bei richtiger Stellung der Ausschnitte der Scheiben ein Spectrum, das in einer Horizontallinie getheilt erschien und dessen obere und untere Hälfte verschieden hell gemacht werden konnten. Die Versuchsanordnung ist übersichtlich dargestellt in Fig. 4, wo a die Lampe, b den Spalt darstellt, von dem die achromatische Linse c in $d$ auf der Scheibe e ein Bild entwirft: $d$ ist fälschlich (der Uebersicht zu Liebe) von e entfernt geeichnet. In e bedeuten die einzelnen Striche (von links nach rechts): Metallscheibe, welche die Kreistheilung trägt, grosser Papiersector, kleiner Papiersector, Metallplatte zur Fixirung der Papiersectoren. Hinter der Scheibe f steht dieCollimatorlinse $g$, welche das Licht parallelstrahlig auf die Prismen $\mathrm{h}$ und $\mathrm{i}$ wirft, von diesem dispergirt fällt es endlich in das auf unendlich eingestellte Fernrohr K. In das Ocular des letzteren ist ein Diaphragma 
eingelegt, durch welches eine bestimimte Farbe isolirt wird. Homogeneität der gesehenen Farbe und möglichste Breite dieses Stückes liessen bald eine passende, mittlere Weite des Diaphragmas finden. Die nachstehenden Versuche sind mit einem solchen angestellt, das mir unter einem Gesichtswinkel von etwas über $3^{0}$ erschien. Das Variiren der Dauer des Eindrucks geschah so, dass für den Einzelversuch die grossen Sectoren von einander um eine bestimmte Zahl von Graden entfernt wurden. Innerhalb des so gebildeten Ausschnittes von bestimmter Grösse wurde dann der kleinere Sector solange verschoben, bis die zwischen ihm und dem jetzt feststehenden Rande des grossen Sectors freigelassene Oeffnung der Zeit entspràch, die gesucht wurde. Es wurde also (Fig. 3) für den Einzelversuch die Zeit a c, a g, a k u. s. f. constant erhalten und die Zeiten b c, $\mathrm{f} g, h \mathrm{k}$ u. s. f. in der Weise gesucht, dass die Punkte $b, f, h$ die variabeln waren. Es erhielt nach einander der Rand des kleinen Sectors Stellung'en, die den Punkten $h^{\prime}$ und $h^{\prime \prime}$ entsprachen und von diesen Punkten aus wurde dann jedesmal im Versuch gegen $\mathbf{h}$ hin der Sector verschoben, bezw. dieser Punkt noch etwas überschritten. Das Mittel aus diesen beiden Beobachtungen gab den Punkt h, d. i. die Zeit h k.

Um an der Zeichnung dies nochmals durch ein Beispiel zu erklären, so würde bei der Art, wie dort die Papiersectoren angeordnet sind und bei der Art der Rotation, wie der eingezeichnete Pfeil andeutet, im Augenblicke, wo $\alpha \beta$ den höchsten Punkt der Scheibe B einnimmt, bezw. gerade überschritten hat, die schwächere Lichtquelle auf das Auge einwirken können und deren Einwirkung durch die Dauer des Vorübergangs $\beta \vartheta$ währen: im Momente, wo $o \lambda$ den höchsten Stand erreicht, bezw. eben überschritten hat, wird nun auch die stärkere Lichtquelle aufgedeckt und deren Einwirkung auf das Auge während der Dauer des Vorübergangs der Strecke $\lambda \mu$ währen. Beide werden gleichzeitig durch den Rand des grösseren Papiersectors wieder zugedeckt. Es muss nun im Einzelversuch - immer natürlich bei constanter Rotationsgeschwindigkeit des Apparates - der Stand des Randes des kleineren Papiersectors $o \lambda$ durch Verschieben gegen $\alpha$ oder gegen $\mu$ hin gesucht werden, bei welchem die stärkere Lichtquelle so lange auf das Auge einwirkt, dass beim Abschneiden der ganzen Erscheinung durch das Vortreten des Sectorrandes $9 \mu$ die durch beide verschieden starke Lichtquellen auf das Auge hervorgebrachten Eindrüicke eben genau gleich sind, 
Es ist bei diesen Versuchen die Voraussetzung gemacht, dass die Erregbarkeit einer bestimmten Netzhautstelle durch die Erregung einer benachbarten nicht verändert wird. Diese Annahme, welche an sich sehr willkürlich, wohl kaum in der ausgesprochenen Allgemeinheit zugestanden würde, erscheint gerechtfertigt bei den sehr kleinen Zeitintervallen, die in den von mir angestellten Versuchen awischen den Anfängen der Reizungen beider Netzhautstellen liegen.

Ich will hier gleich noch eine weitere Unregelmässigkeit meiner Versuche erwähnen, die darin besteht, dass bei der Beleuchtung nur der einen Hälfte des Gesichtsfeldes im Fernrohr die andere Hälfte nicht absolut dunkel war, sondern durch in den Prismen diffus zerstreutes Licht eine Spur von Belichtung zeigte. Es begann drum die Reizung der zweiten Netzhautstelle nicht von Null, sondern von einem sehr geringen Werthe. Es war derselbe sehr niedrig gegenüber den als Versuchsobjecte gebrauchten Helligkeiten. Zu beseitigen wäre dieser Missstand nur, wenn man die Scheibe mit den Papiersectoren durch einen Schlitz im Oculare des Beobachtungsfernrohres selbst rotiren liesse.

Für die Reinheit der Versuche ist es natürlich von hohem Werthe, dass das Auf- und Zudecken der Lichtquelle möglichst momentan geschieht, und dass dem Einzelversuche direct vorangeht und nachfolgt möglichst vollständigé Dunkelheit. Beiden Bedingungen ist in der oben beschriebenen Versuchsanordnung sehr vollkommen entsprochen.

Die Resultate, die ich bei zahlreichen Versuchen erhielt, sind die folgenden:

I. Die verschiedenen Theile des Spectrum brauchen verschiedene Zeit, um das Maximum der Erregung hervorzubringen, und zwar ist diese Zeit für Roth unter allen Umständen die kürzeste, dann folgt Blau und Grün, von denen bei gleicher (subjectiver) Helligkeit Blau den Vorrang hat.

Ich gebe als Beleg hierfür die nachfolgenden Tabellen.

Zeit die nothwendig ist für verschiedene Theile des Spectrum, um (bei gleicher Spaltweite) das Maximum von Erregung hervorzubringen:

$$
\begin{array}{ccc}
\text { Roth } & \text { Grün } & \text { Blau } \\
0,0573 \text { Sec. } & 0,0971 \text { Sec. } & 0,1018 \text { Sec. }
\end{array}
$$


Beziehe ich die Zahlen auf ungefähr gleiche Helligkeit, so ergibt sich:

Zeit, die nothwendig ist, damit verschiedenfarbiges (ungefähr) gleich helles Licht das Maximum der Erregung hervorbringt: $\begin{array}{ccc}\text { Roth } & \text { Grün } & \text { Blau } \\ 0,0573 \text { Sec. } & 0,133 \text { Sec. } & 0,0916 \text { Sec. }\end{array}$

Ich habe diese letztere Zusammenstellung nur nach beiläufigen Schätzungen gemacht und dabei angenommen, dass in dem vom Lichte der Petroleumlampe erzeugten Spectrum Grün doppelt so hell als Roth und viermal so hell als Blau sei. Ich habe der Uebersichtlichkeit $\mathrm{zu}$ Liebe ein so einfaches Verhältniss aufgestellt und dürfte dabei die obige Annahme vielleicht für Roth etwas zu hoch sein, da Roth in dem gelblich aussehenden Lichte einer Petroleumlampe in bedeutender Stärke enthalten ist: für Blau dagegen noch eher etwas zu nieder, weil an sich wenig Blau in dem gelblichen Petroleumlichte enthalten ist und der nach kurzem Gebrauche immer etwas gelblich aussehende Schwefelkolenstoff blaues Licht absorbirt. Auch haben die obigen Annahmen nur für eine ganz bestimmte Beleuchtungsintensität Gültigkeit, weil, wie $\mathrm{Helmholtz}{ }^{1}$ ) gezeigt hat und wie ich auch im Verlaufe dieser Versuche gelegentlich bemerken konnte, mit Aenderung der objectiven Helligkeit des ganzen Spectrum die Empfindungsstärken für die verschiedenen Theile desselben nicht in gleichem Verhältnisse sich ändern. Die obige Annahme ist gemacht für ein Helligkeitsverhältniss, wo der Spalt für Grün durch $1 / 4$ Umdrehung einer ganzen Windung der Mikrometerschraube, für Roth durch $1 / 2$ und für Blau durch 1 Umdrehung geöffnet war. Die Oeffnung des Spaltes durch Umdrehung einer ganzen Windung der Mikrometerschraube ist gleich 0,354 $\mathrm{Mm}$.

Die obigen Zahlen stehen in directem Widerspruche mit Angaben von Lamansky ${ }^{2}$ ), die er bei Mittheilung anderer Versuchsresultate gelegentlich macht und wornach die Zeiten, die verschiedenfarbiges Licht gebraucht, um eine bestimmte Netzhautstelle in das Maximum von Erregung zu versetzen, nur abhängig sind von der (subjectiven) Helligkeit dieses Lichtes in dem Sinne des sub II ausgesprochenen Satzes, wonach also beispielsweise im gleichen Spectrum das (subjectiv) weniger helle Roth längere Zeit gebrauchte,

1) Physiologische Optik pag. 318.

2) Ueber die Grenzen der Empfindlichkeit des Auges für Spectralfarben, Gräfe's Archiv XVII, 1, pag. 123. 
als das hellere Grün u. s. f. Ob diese Verschiedenheit der Resultate auf Verschiedenheit der Methode oder der angewandten Lichtquelle (Lamansky benutzte Pigmente) oder worauf sonst beruht, vermag ich nicht anzugeben.

Ich habe in Tabelle 1 die Resultate der von mir angestellten Versuche zusammengestellt. Die den Columnen überschriebenen Zahlen $1 / 4,1 / 2,1$ bedeuten das Verhältniss der zu diesen Versuchen verwendeten Helligkeiten: die in den Columnen selbst stehenden Zahlen bedeuten Secunden und sind gleich der Zeit, die zu maximaler Erregung nothwendig ist. In Tabelle 2, 3 und 4 habe ich probeweise einige Originalversuche in extenso zur Erlüuterung und Prüfung der Methode angefiihrt. In der ersten Columne dieser Tabellen bedeuten die Zahlen die Oeffnung des Spaltes, ausgedrückt in Umdrehungen der Mikrometerschraube, die zweite Columne enthält die Zeiten, durch welche jeweilig die geringeren Helligkeiten auf das Auge einwirkten: die dritte und vierte Columne enthält die direct durch den Versuch gewonnenen Stellungen des kleineren Papiersectors, von welchen Columne 5 das Mittel angibt. Die Zahlen der Columne 6 sind die Differenzen der in der zweiten und fünften Columne enthaltenen Zahlen und sind direct den Zeiten proportional, die die grössere Helligkeit zur Hervorbringung gleicher Erregung gebraucht. Aus der in Columne 7 angegebenen Rotationsgeschwindigkeit können diese Zeiten leicht in absolutem Maasse berechnet werden. Ausrufezeichen in Columne 3 und 4 bedeuten, dass der Gleichheitspunkt auffallend überschritten war.

Zu Tabelle 1 noch einige Erläuterungen. Die Versuche sind an. verschiedenen Tagen und selbstverstïndlich vollständig unabhängig von einander und im Einzelnen angestellt. Die Uebereinstimmung unter denselben erscheint mir so vollständig, als dies bei den Fehlerquellen solcher subjectiven Versuche nur zu erwarten ist. Ls sind nämlich dieselben ziemlich anstrengend und langwierig und verlangen viel Vorübung, um bei der immer wieder im Verlaufe des Einzelversuchs anzustellenden Controle Uebereinstimmung zu geben. Die Fehlerquellen liegen vor Allem in dem jeweiligen Erregbarkeitszustande des Auges. Ich beobachtete im (fast absolut) dunkeln Raume und immer erst dann, wenn sich das Auge vollständig nadaptirt ( $\left.{ }^{1}\right)$ hatte. Trotzdem ist natürlich doch noch in Folge vor-

1) Aubert, Physiologie der Netzhaut, pag. 25. 
ausgegangener Anstrengung oder in psychischen Zuständen (Aufmerksamkeit etc.) die Wahrscheinlichkeit gegeben, dass der Zustand der Erregbarkeit nicht immer der gleiche war. Alle Versuche sind mit dem linken Auge angestellt, auf dem ich nach gewöhnlicher Bezeichnungsweise ${ }^{-1} / 10$ bis $1 / 11$ myopisch bin. Merkwürdig ist, dass ich am sichersten immer Nachmittags beobachtete: ich konnte kaum jemals an Vormittagen zu gut gelungenen Versuchen kommen. Eine weitere Fehlerquelle liegt darin, dass nicht immer zur Erzeugung der gleichen Empfindung genau der gleiche Theil des Spectrum eingestellt war, was bei dem Spectrum des Petroleumlichtes, wo die orientirenden Linien fehlen, nicht zu erreichen ist. Ich versäumte von Anfang an, da ich den Versuchen die wirklich erreichte Genauigkeit nicht zutraute, dies in anderer Weise zu Stande zu bringen, was ja durch Visirzeichen, die an dem Fernrohre und an der gegenüberliegenden Wand angebracht waren, bei definitiv feststehender Versuchsanordnung in toto leicht gelungen wäre. Natürlich wurde immer aus dem Gedächtnisse wieder der gleiche Farbenton eingestellt, was auch wohl sehr genau gelingt. Es mag vielleicht aus dem eben Gesagten kommen, dass die bessere Uebereinstimmung der mit Roth angestellten Versuche unter einander darin ihren Grund hat, weil die rothe Zohne des Brechungsspectrum die relativ schmälste ist und darum immer genauer der gleiche Theil wieder eingestellt werden kann, als bei Grün und Blau dies möglich ist. Für letztere beide Farben mag die Unregelmässigkeit, die sich in Tabelle 1 zeigt, dass nämlich die späteren Versuche im Allgemeinen eine immer grössere Dauer aufzuweisen haben, darauf beruhen, dass der Schwefelkohlenstoff im Prisma nach und nach sich gelb färbte, wodurch natürlich das grüne und vor Allem das blaue Licht geschwächt wurde. Es würde sich diese Abweichung aus dem sub II Angegebenen erklären. Auch sind Unregelmässigkeiten und Verschiedenheiten in der Versuchsanordnung nicht ganz zu vermeiden. Finer weiteren Ausdehnung der Versuche setzt der Zustand der Augen, welche durch dieselben in gar nicht zu vernachlässigender Weise angegriffen werden, bald selbst eine Grenze.

II. Für die gleiche Farbe gilt der Satz, dass die grössere Helligkeit in kürzererZeit das ihr zukommende Maximum von Erregung hervorbringt als die kleinere.

Als Beweis führe ich Mittelzahlen aus Tabelle 1 an. 
Zeit, die bei verschiedener Helligkeit $\mathrm{zu}$ maximaler Erregung nothwendig ist:

Farbe: Helligkeitsverhältniss:

\begin{tabular}{|c|c|c|c|c|}
\hline & 1 & 2 & & 4 \\
\hline Roth & 0,071 Sec. & 0,0573 & Sec. & - \\
\hline Grï & 0,133 & 0,097 & $D$ & 0,0699 \\
\hline la & - & 0,102 & $D$ & 0,0916 \\
\hline
\end{tabular}

Es hat diesen Satz Exner für weisses Licht zuerst experimentell bewiesen und aus einer grösseren Beobachtungsreihe den Satz abgeleitet, dass die Zeiten, die zu maximaler Erregung nothwendig sind, in arithmetischer Progression zunehmen, wenn die zugehörigen Intensitäten in geometrischer abnehmen.

Ich habe den obigen allgemeinen Satz auch für weisses Licht durch das Experiment bestätigt gefunden. Dagegen habe ich es nicht versucht, die gesetzmässige Beziehung, die Exner aufstellt, auch für farbiges Licht zu erweisen. Einmal erscheinen mir die bei der Methode möglichen Fehlergrenzen zu weit, um aus darnach gewonnenen Zahlen eine solche Beziehung abzuleiten; dann sind grössere Helligkeiten spectralen Lichtes, wegen der starken Blendung, die das adaptirte Auge durch sie erfährt, kaum anzuwenden; endlich steht auch ein principielles Bedenken entgegen. Es kann nämlich nothwendigerweise eine solche Beziehung zwischen Intensität der Lichtquelle und der zu maximaler Erregung nothwendigen Zeit nur innerhalb gewisser, und zwar ziemlich enger Grenzen Geltung haben, beziehungsweise erwiesen werden. Betrachtet man z. B. die Zahlen der zweiten Tabelle, die Exner auf Seite 616 seiner Abhandlung angibt, so würden sich bei Fortsetzung der Reihe schon für die nächsten Glieder Werthe ergeben, die um Null oder selbst unter Null fallen. Nun brauchen aber auch sehr grosse Zeiten bis zu maximaler Erregung relativ grosse, messbare Zeiten. Nach der Seite der wachsenden Intensitäten muss sich also wohl die fragliche Zeit bald einem Grenzwerthe nähern. Nach der Seite der abnehmenden Intensitäten scheint mir der experimentelle Nachweis dieses Gesetzes bald unmöglich zu werden, weil ỉmmér bei derlei Versuchen der Grundgedanke der Methode die Unterscheidung, beziehungsweise Gleichmachung der Erregungen verschiedener Netzhautstellen sein wird und für kleine Helligkeiten experimentell festgestellt ist ${ }^{1}$,

1) Aubert, Physiologie der Netzhaut, pag. 62 u. ff. 
dass die Unterschiedsempfindlichkeit sehr gering und unzuverlässig wird.

III. Die Form des Anklingens der Erregung lässt sich unter gewissen, zulässigen Annahmen aus den von mir angestellten Versuchen construiren. Diese Annahmen sind: a) dass gleich mit Beginn der Reizung die Erregung beginnt, und b) dass der erste Theil der Erregungscurve geradlinig ansteigt. Die erste dieser Fragen ist schon von Exner (a. a. 0. pag. 622) experimentell und im Sinne der obigen Forderung bejahend entschieden. Er fand, dass schon bei 0,0001 Sec. Einwirkungsdauer eine deutliche Empfindung zu Stande kam. In Versuchen, die ich sub V besprechen werde, fand ich ganz ähnliche Zeiten auch für homogenes Licht. Sollte wirklich bei noch kürzerer Einwirkung eine Empfindung nicht mehr zu Stande kommen, so würden solche Zeiten weit in die Fehlergrenzen meiner Versuche fallen. Die zweite der obigen Annahmen wird durch den Versuch mit einer Genauigkeit erwiesen, die ihre Anwendung gestattet. Wenn man nämlich für einen bestimmten Theil der ansteigenden Erregungscurve, der übrigens ganz im Anfange derselben gelegen sein muss, die Zeit sucht, die eine zweite viel grössere Helligkeit braucht, um den gleich hohen Erregungszustand in einer anliegenden Netzhautstelle hervorzubringen, so wird für diese letztere Helligkeit die Zeit doppelt so gross gefunden, wenn man von der Erregungscurve ein doppelt so grosses Stück wie im ersten Versuche nimmt, d. h. die Dauer der Einwirkung der schwächeren Helligkeit verdoppelt. Wenn also in Fig. 3 das Stück a $\mathrm{z}$ ganz im Beginne der Erregungscurve liegt, so ist, wenn a c $=1 / 2 \mathrm{ag}$, auch immer durch den Versuch festzustellen, dass $b c=1 / 2 \mathrm{fg}$. Es ist indess die Genauigkeit dieser Beobachtungen nicht so gross, dass dadurch in Wahrheit ein geradliniges Ansteigen der Erregungscurve bewiesen werden kann. Es ist vielmehr wahrscheinlich, dass die Neigung derselben zur Abscissenaxe sich stetig ändert. Nur ist diese Aenderung im Beginne so gering, dass trotz besserer Ueberzeugung die obige Annahme gestattet ist. Es ergibt sich nun durch den Versuch, dass dieses - annäherungsweise geradlinige Ansteigen der Erregungscurve ein um so grösseres Stück der ganzen Curve einnimmt, je grösser die Helligkeit ist, der diese Erregungscurve zugehört. Aus dieser Beobachtung leitet sich erst die Berechtigung $a b$, den Zeiten, die für jeden Einzelversuch die zweite grössere Helligkeit bedarf, um gleich grosse Erregung zu 
bedingen, direct proportional zu setzen die während derselben hervorgebrachten Erregungen, d. h. man darf annehmen, dass in Fig. 3 die Stücke der zweiten Erregungscurve, bs, fz, hx, iy etc. wirklich geradlinig ansteigen. Als Gegensatz dazu ergibt sich, dass die Erregungscurven schwächerer Helligkeiten schon bald ihren Verlauf so ändern, dass sie immer stärker gegen die Abscissenaxe sich neigen. Ich glaube beobachtet zu haben, dass das Abfallen der Erregung um so steiler ist, je höher die Erregung war und je kürzer die Zeit, die es bis zur Erreichung des Maximums bedurfte, so dass also beispielsweise bei gleich hellem Roth und Grün die Erregungscurve für Roth rascher abfiele als die für Grün. Doch will ich die letztere Behauptung nicht als sicher hinstellen, da die Beobachtungen, die spätere Punkte der Erregungscurve darstellen, sehr mühevoll und darum wenig zahlreich sind, wegen der starken Blendung, die das Auge von den um den Maximalwerth herumliegenden Helligkeiten erfährt und wegen der Störung und Unsicherheit, die durch das ungleichzeitige Erscheinen beider Lichtquellen bedingt ist.

Ich habe in Fig. 5 einige Curven für verschiedenfarbiges Licht und verschiedene Reizintensitäten nach Zahlen, die direct durch den Versuch gewonnen sind, construirt.

IV. Es ändert sich mit der Helligkeit auch Farbenton und Sättigung. Ich habe schon oben (pag. 198) auf die gegenseitige Abhängigkeit dieser drei Grössen hingewiesen.

Es ist dies eine schon von $\mathrm{H} \mathrm{elm} \mathrm{hol} \mathrm{tz} \mathrm{experimentell} \mathrm{festgestellte}$ Sache, die sich in dem Satze zusammenfassen lässt, dass mit zunehmender Helligkeit farbigen Lichtes die dadurch hervorgerufenen Empfindungen dem Weiss zustreben. Ich konnte im Verlaufe meiner Versuche dies immer wieder beobachten, wobei mir auch sehr deutlich auffiel, dass Blau ohne Aenderung seines Farbentons in Weiss übergeht, während Grün und Roth durch Gelb sich der Empfindung Weiss nähern.

Es ist aus diesem Grunde misslich den Begriff der Helligkeit farbigen Lichtes zu definiren. Dieselbe ist nach der unsere Ansichten über das Zustandekommen von Farbenempfindungen überhaupt beherrschenden Hypothese die Summe von im Bewusstsein nicht getrennten Erregungen verschiedenartiger Nervenendapparate. Wir werden also von zwei durch verschiedene Intensitäten gleichartigen homogenen Lichtes erzeugten Empfindungen diejenige für die stärkere erklären - sie für die hellere halten, in der eventuell die Stärke der specifischen Farbenempfindung die geringere ist. 
Es drücken darum die in Fig. 5 verzeichneten Curven nicht etwa den zeitlichen Verlauf der Erregung der rothes, grünes, blaues Licht percipirenden Nervenendapparate aus, sondern es sind diese der Ausdruck sehr complicirter Vorgänge und es ist nur der beiläufige Schluss erlaubt, dass - mit beständiger Voraussetzung der Richtigkeit der Young'schen Theorie natürlich - die eigentlichen Erregungscurven für die Roth, Grün, Blau empfindenden Fasern ähnlich aussehen, wahrscheinlich noch steiler ansteigen werden.

Es war mir nun sehr auffallend zu bemerken, dass die Intensitäten farbigen lichtes, die jch untersuchte, bei kurzer Dauer der Einwirkung (ebenso lange, wie zu maximaler Erregung nothwendig ist) so ungemein hohe Erregungswerthe hervorbrachten und eine sehr weitgehende Aenderung des Farbentons zeigten, während die gleichen Theile des Spectrums bei derselben (objectiven) Helligkeit bei dauernder Betrachtung viel weniger intensiv das Auge erregten und auch immer durchaus den Eindruck der eben diesem Theil des Spectrums zukommenden Farbe in grosser Sättigung machten. So machte z. B. die Helligkeit, welche die auf Tabelle 1 unter Grün Columne $1 / 2$ verzeichneten Versuche ergab, bei dauernder Betrachtung den Eindruck eines angenehmen Grün, beim Abschneidən der Erregung dagegen an dem Zeitpunkte maximaler Erregung den Eindruck eines blendenden Gelb. Natürlich muss bei continuirlichem Betrachten die zeitliche Aufeinanderfolge der einem bestimmten Reize zugehörigen Empfindungsqualitäten die genau gleiche sein, wie bei nur kurzer Dauer des Reizes. Wir werden uns nur der im Beginne der Erregungscurve liegenden Empfindungsqualitäten nicht bewusst und was wir als die einer bestimmten homogenen Lichtart entsprechende Empfindung bezeichnen, ist eine Empfindung, die schon weit auf dem absteigenden Theile der Erregungscurve liegt. Es würde also z. B. für grünes Licht, wofür diese Erscheinung am frappantesten ist, die Aufeinanderfolge der verschiedenen Empfindungsqualitäten durch Fig. 6 dargestellt werden können, wo die Punkte a,a,a der Empfindung Grün, b gelblich Grün, c Gelb, d gelblich Weiss, f,f,f Grün entsprechen. Es ist wohl auch daraus, dass die Aenderung der Empfindung so rasch verläuft und bei continuirlichem Betrachten gar nicht beachtet wird, der Schluss erlaubt, dass der Anfang des abfallenden Theiles der Erregungscurve viel rascher sinkt als spätere Stücke desselben Theiles, wie ich in Fig. 6 dies hypothetisch gezeichnet habe. - Es nöthigt die eben beschriebene Erscheinung zu 
der Annahme, dass für die Auffassung von Lrregungszuständen unserer Nervenapparate die Dauer derselben einen so wesentlichen Einfluss hat, dass bei längerer Dauer die weniger intensive Erregung die stärkere, aber kurz dauernde im Bewusstsein verdrängt.

Den Grund für den Verlauf dieser Erscheinung wird man in dem Vermögen der Netzhaut suchen müssen, sich verschiedenen Helligkeiten zu adaptiren; dieses beruht einmal auf dem Zustandekommen der reflectorischen Pupillenverengerung, dann allerdings auch auf Aenderungen des Erregungszustandes der Netzhautelemente selbst (Ermüdung und Erholung). Die Versuche sind im Dunkeln angestellt, wo das Auge sehr empfindlich und die Pupille bis zum Maximum erweitert ist. Da aber direct der Fläche der Pupillenöffnung proportional bei sonst günstigen Bedingungen die Summe der auf das einzelne Retina-Element fallenden Lichtstrahlen sein wird, so muss das sehr empfindliche Auge bei plötzlich beginnender Reizung, selbst durch eine geringere Helligkeit, eine sehr starke Erregung erfahren, die reflectorisch eine Irisverengerung auslöst. Nur darauf beziehe ich das auch in Fig. 5 angedeutete und durch die obigen Erscheinungen für Fig. 6 postulirte, so ungemein rasche Abfallen der Erregungscurve. Allerdings sind die Zeiten, um die es sich hier handelt, relativ kurz gegenüber denen, welche $\operatorname{Exner}^{1}$ ) gefunden hat für das Zustandekommen von Reflexen. Doch ist diese Differenz nicht so gross, dass man nicht das angezogene Moment zur verwendeten Erklärung benutzen könnte. Inwieweit die Ermüdung dabei als Componente betheiligt ist, lässt sịch nicht bestimmen. Indess ist es höchst wahrscheinlich, dass ein so rasches Sinken der Erregungscurve dadurch nicht bedingt sein kann. Will man die obige Erscheinung auf andere Weise, durch die Hypothese verschiedenen zeitlichen Verlaufes der Erregungen der drei Fasergattungen der Netzhaut durch verschiedenfarbiges Licht erklären, so werden so complicirte Annahmen nothwendig, dass man von so gezwungener Erklärung bald absteht.

Ich glaube, dass dieses Adaptationsvermögen der Netzhant bei der subjectiven Vergleichung verschiedener Helligkeiten wohl zu beachten ist, und dass man bisher bei der Discussion der Lehre von der Empfindungsstärke den durch die Reizgrösse selbst beding-

1) Ueber Reflexzeit und Rückenmarksleitung. Dies Archiv VIII, 10. Heft, pag. $526 \mathrm{ff}$. 
ten jeweiligen Erregbarkeitszustand der Netzhaut weniger berücksichtigt hat, als dies nothwendig erscheint.

Die Annahme, dass diese reflectorische Pupillenverengerung schon in kürzerer Zeit zu Stande komme, als überhaupt eine bestimmte Lichtintensität das Maximum von Erregung gesetzt habe, durch welche Annahme natürlich der Werth der unter I und II beschriebenen Versuche sehr zweifelhaft wäre, kann man bei der Kürze der in Frage kommenden Zeiten direct zurückweisen. Beweis für die Richtigkeit dessen ist eben die in diesem Abschnitte besthriebene Erscheinung.

V. Bei sehr kurz dauernder Einwirkung homogenen Lichtes auf das Auge ändert sich ebenfalls der Farbenton, und zwar in der Weise, dass das ganze Spectrum jetzt nur melur in zwei Theile getheilt erscheint, deren einer den Eindruck Roth, der andere den von Blau macht. Am frappantesten ist diese Erscheinung, wenn man in das Fernrohr die Mitte des Spectrums einstellt, von röthlich Gelb bis grünlich Gelb: es entsteht dann deutlich (bei nur hinreichend kurzer Dauer) der Eindruck, dass das Gesichtsfeld links schwach roth, rechts blau erscheint. Natürlich ist der Farbeneindruck nicht glänzend, aber doch so deutlich, dass man eine genau charakterisirbare Farbenempfindung erhält. Es scheint bei diesen Versuchen darauf anzukommen, dass die Erregung einen bestimmten Werth erlangt, gleichviel ob dies durch eine gewisse Dauer der Einwirkung der Lichtquelle oder bei geringerer Dauer durch Vermehrung der Reizintensität geschieht ${ }^{1}$ ). Macht man die Erregung durch Abnahme dieser beiden Factoren noch geringer, so kommt man bei einem Punkte an, wo zwar noch Lichtempfindung, aber keine Farbenempfindung mehr zu Stande kommt. Nur das rothe Ende des Spectrums bedingt bei mir, wenn überhaupt eine Empfindung, dann auch sofort eine, und zwar gesättigte Farbenempfindung. Es sind also die Resultate dieser Beobachtungen in Kürze die folgenden. Der sogen. rothe Theil des Spectrums erregt bei sehr kurzer Dauer der Einwirkung auf das Auge keine Empfindung; bei

1) Die dritte hier noch denkbare Möglichkeit hat Aubert (Physiologie der Netzhant pag. 128) durch den Versuch geprüft; er beobachtete Pigmente bei abnehmender Beleuchtungsintensität: die Uebereinstimmung der Resultate ist sehr vollständig. 
allmählicher Vergrösserung der Erregung (durch Dauer der Einwirkung oder Vergrösserung der Intensität) entsteht sofort die Empfindung Roth. Der sogen. grüne Theil des Spectrums bringt bei minimaler Einwirkung eine Lichtempfindung überhaupt, bei Vergrösserung der Erregung die Empfindung Blau, bei noch weitergehender Erregung die Empfindung Grün hervor. Das rechte Ende des Spectrums erscheint bei solcher stufenweisen Verfolgung zuerst als Lichtschein, dann bedingt es die Empfindung Blau.

Ich habe in der Tabelle 5 eine solche Originalbeobachtung in extenso mitgetheilt und absichtlich eine solche gewählt, die ich mit einem in physikalischen Dingen absolut unerfahrenen Beobachter angestellt habe, um jede Möglichkeit einer Selbsttäuschung, die vielleicht hier durch erkannte Stellung des Fernrohres zum Prisma etc. denkbar wäre, auszuschliessen. $E_{s}$ weichen die von diesem Beobachter gemachten Angaben von denen, die ich von andern Beobachtern und mir selbst erhielt, darin ab, dass er bei Einstellung der Mitte des Spectrums die stattgehabte Empfindung als "links Gelb, rechts Blau “ beschrieb, während sie uns Anderen als "links Roth, rechts Blau « vorkam.

Man wird bei einiger Ueberlegung einen inneren Zusammenhang zwischen den in diesem Abschnitte mitgetheilten Erscheinungen und dem unter I Angegebenen finden. Weitere theoretische Speculationen will ich indess vermeiden.

VI. Ich füge hier im Anhange noch einige Versuche an, die ich gegen das Ende meiner Untersuchungen angestellt habe.

Wenn nämlich, wie bei den vorliegenden Bedingungen innerhalb der unvermeidlichen Fehlergrenzen bewiesen ist, wirklich der Anfangstheil der Erregungscurve einer grossen Helligkeit geradlinig ansteigt und gleich mit Anfang der Reizung auch die Erregung beginnt, so entsprechen proportionalen Stücken dieser Curve auch wirklich proportionale Erregungen. Wenn also Anfangsstücke einer solchen Curve Erregungswerthe darstellen, welche den von geringeren Helligkeiten hervorgebrachten Maximis der Erregung gleich sind, so hat man damit ein Maass, um verschiedene Reizintensitäten auf die Grösse der von ihnen bedingten Erregungen zu untersuchen. Ich führte Versuche in der Weise aus, dass ich Reizintensitäten, die selbst eine arithmetische Reihe bilden, untersuchte, indem ich die von ihnen bedingten maximalen Erregungen verglich mit der Erregung durch eine zweite viel grössere Helligkeit und nun 
zusah, durch wie lange Zeiten ich diese zweite Helligkeit jedesmal einwirken lassen musste, um gleich hohe Frregungswerthe, wie sie die geringeren Helligkeiten nach einander setzten, hervorzubringen. Ich habe in Tabelle 6 ein Beispiel der auf solche Weise gewonnenen Zahlen gegeben. Der Umfang der von mir angestellten Versuche, deren weitere Ausdehnung der Zustand meiner Augen verbietet, ist zu gering, als dass daraus Schlüsse zu ziehen erlaubt wäre. Da indess noch eine grössere Empfindlichkeit bei dergleichen Versuchen zu erreichen ist, als ich sie angewendet habe, so wäre es vielleicht durch sehr weite Ausdehnung sogar möglich, eine ganz directe experimentelle Ermittelung der Beziehung zwischen Reiz- und Empfindungsgrösse anzustreben.

Tabelle 1.

1 Umdrehung c. $0,354 \mathrm{Mm}$. Spaltweite.

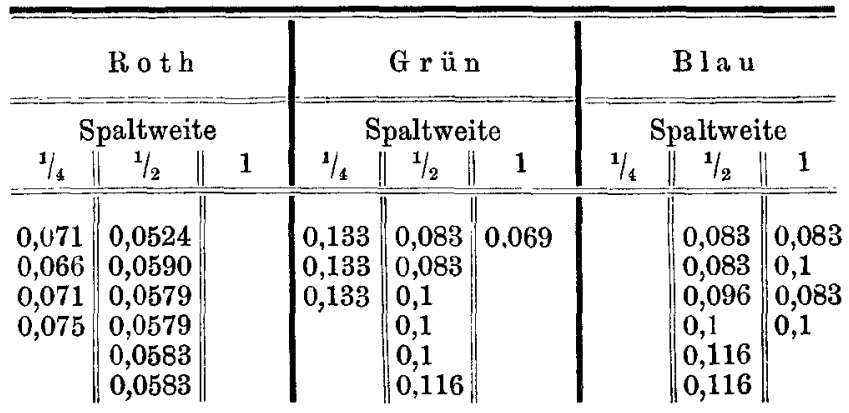

Tabelle 2.

\begin{tabular}{|c|c|c|c|c|c|c|}
\hline $\begin{array}{c}\text { Farbe } \\
\text { und } \\
\text { Helligkeit }\end{array}$ & $\begin{array}{c}\text { Oeffnung } \\
\text { d, grossen } \\
\text { Sectoren } \\
\text { von } 0^{\circ} \text { bis }\end{array}$ & \multicolumn{2}{|c|}{\begin{tabular}{|} 
Stand des kleinen \\
Sector - Randes, \\
gesucht von \\
d.kleinen/d.grossen \\
Zahlen her
\end{tabular}} & Mittel & $\begin{array}{l}\text { Diffe- } \\
\text { renz }\end{array}$ & $\begin{array}{c}\text { Rotationsgeschwin- } \\
\text { digkeit }\end{array}$ \\
\hline Roth $1 / 2$ & $\begin{array}{r}20 \\
40 \\
60 \\
80 \\
100 \\
120 \\
140\end{array}$ & $\begin{array}{l}16,8 \\
33 \\
52 \\
71 \\
91,1 \\
111,3 \\
132\end{array}$ & $\begin{array}{c}16,2 \\
32,7 \\
52,5 \\
71 \\
90,8 \\
111 \\
131\end{array}$ & $\begin{array}{c}16,5 \\
32,9 \\
52,2 \\
71 \\
90,9 \\
111,1 \\
131,5\end{array}$ & $\begin{array}{l}3,5 \\
7,1 \\
7,8 \\
9 \\
9,1 \\
8,9 \\
8,5\end{array}$ & $\begin{array}{c}\text { R.-Geschwindigkeit d. } \\
\text { hinteren Scheibe } \\
5,33 \text { Umdreh. in } 1 \text { Sec. } \\
1^{0}={ }^{2}{ }_{1900} \text { Sec. }\end{array}$ \\
\hline Roth $1 / 2$ & $\begin{array}{l}40 \\
60 \\
70 \\
80 \\
90\end{array}$ & $\begin{array}{l}33 \\
51 \\
61 \\
70,8 \\
81\end{array}$ & $\begin{array}{l}32,5 \\
51 \\
60,6 \\
70,3 ! \\
81\end{array}$ & $\begin{array}{l}32,8 \\
51 \\
60,8 \\
70,6 \\
81\end{array}$ & $\begin{array}{l}7,2 \\
9 \\
9,2 \\
9,4 \\
9\end{array}$ & $\begin{array}{c}\text { Geschwindigkeit 3,33 } \\
1^{\circ}=1 / 1200 \text { Sec. }\end{array}$ \\
\hline
\end{tabular}




\begin{tabular}{|c|c|c|c|c|c|c|}
\hline $\begin{array}{c}\text { Farbe } \\
\text { und } \\
\text { Helligkeit }\end{array}$ & $\begin{array}{l}\text { Oeffnung } \\
\text { d. grossen } \\
\text { Sectoren } \\
\text { von } 0^{\circ} \text { bis }\end{array}$ & \multicolumn{2}{|c|}{\begin{tabular}{|l||} 
Stand des kleinen \\
Sector-Randes, \\
gesucht von \\
d.kleinen|d.grossen \\
Zahlen her
\end{tabular}} & Mittel & $\begin{array}{c}\text { Diffe- } \\
\text { renz }\end{array}$ & $\begin{array}{c}\text { Rotationsgeschwin- } \\
\text { digkeit }\end{array}$ \\
\hline Roth $1 / 4$ & $\begin{array}{r}30 \\
60 \\
80 \\
90 \\
100\end{array}$ & $\begin{array}{l}26 \\
52 \\
71 ! \\
81 ! \\
91,5 !\end{array}$ & $\begin{array}{l}25,8 \\
52 \\
70 \\
80 \\
90 !\end{array}$ & $\begin{array}{l}25,9 \\
52 \\
70,5 \\
80,5 \\
90,7\end{array}$ & $\begin{array}{l}4,1 \\
8 \\
9,5 \\
9,5 \\
9,3\end{array}$ & $1^{0}=1 / 1200$ Sec. \\
\hline $\operatorname{Roth} 1 / 4$ & $\begin{array}{l}30 \\
60 \\
80 \\
90\end{array}$ & $\begin{array}{l}25,4 \\
50 \\
70 ! \\
79,8\end{array}$ & $\begin{array}{l}25,1 \\
51 \\
69,8 \\
79,5\end{array}$ & $\begin{array}{l}25,2 \\
50,5 \\
69,8 \\
79,7\end{array}$ & $\begin{array}{r}4,8 \\
9,5 \\
10,2 \\
10,3\end{array}$ & $1^{0}=1 / 1200$ Sec. \\
\hline
\end{tabular}

Tabelle 3.

\begin{tabular}{|c|c|c|c|c|c|c|}
\hline $\begin{array}{c}\text { Farbe } \\
\text { und } \\
\text { Helligkeit }\end{array}$ & $\mid \begin{array}{l}\text { Oeffnung } \\
\text { d. grossen } \\
\text { Sectoren } \\
\text { von } 0^{\circ} \text { bis }\end{array}$ & \multicolumn{2}{|c|}{$\mid$\begin{tabular}{c|} 
Stand des kleinen \\
Sector-Randes, \\
gefunden von \\
d.kleinen $\mid$ d.grossen \\
Zahlen her
\end{tabular}} & Mittel & $\begin{array}{c}\text { Diffe- } \\
\text { renz }\end{array}$ & $\begin{array}{c}\text { Rotationsgeschwin- } \\
\text { digkeit }\end{array}$ \\
\hline Grün $1 / 4$ & $\begin{array}{r}40 \\
80 \\
120 \\
140 \\
160 \\
180 \\
200\end{array}$ & $\begin{array}{l}32 \\
65 \\
101,5 \\
120 \\
137 ! \\
160 \\
181\end{array}$ & $\begin{array}{c}32 \\
67 \\
102 \\
121 \\
138 ! \\
160,5 \\
180,8\end{array}$ & $\begin{array}{c}32 \\
66 \\
101,8 \\
120,5 \\
137,5 \\
160,2 \\
180,9\end{array}$ & $\begin{array}{c}8 \\
14 \\
18,2 \\
19,5 \\
22,5 \\
19,8 \\
19,1\end{array}$ & $\begin{array}{l}3,33 \text { in } 1 \text { Sec. } \\
1^{0}=1 / 1200 \text { Sec. }\end{array}$ \\
\hline Grün $1 / 2$ & $\begin{array}{r}40 \\
80 \\
120 \\
140 \\
120 \\
170\end{array}$ & $\begin{array}{l}30,3 \\
65 \\
102,3 ! \\
122 \\
132 \\
153\end{array}$ & $\begin{array}{c}31 \\
64,8 \\
101 \\
122 \\
132 \\
152 !\end{array}$ & $\begin{array}{c}36,6 \\
64,9 \\
101,6 \\
122 \\
132 \\
152,5\end{array}$ & $\begin{array}{l}9,4 \\
15,1 \\
18,4 \\
18 \\
18 \\
17,5\end{array}$ & $\begin{array}{c}\text { Rot.-Geschwindigk. } \\
3,33 \text { in } 1 \text { Sec. } \\
1^{\circ}=1 / 1206 \text { Sec. }\end{array}$ \\
\hline Grün $1 / 2$ & $\begin{array}{r}40 \\
80 \\
120 \\
160 \\
200\end{array}$ & $\begin{array}{l}35 \\
71,9 \\
109 \\
149,2 \\
190\end{array}$ & $\begin{array}{l}35 \\
71,6 \\
109,2 \\
149,8 \\
190\end{array}$ & $\begin{array}{c}35 \\
71,7 \\
109,1 \\
149,6 \\
190\end{array}$ & $\begin{array}{c}5 \\
8,3 \\
10,9 \\
10,4 \\
10\end{array}$ & $\begin{array}{c}\text { Rot. } 4,3 \text { in } 1 \text { Sec. } \\
1^{0}=1 / 1550 \text { Sec. }\end{array}$ \\
\hline Grün 1 & $\begin{array}{r}40 \\
80 \\
100 \\
110 \\
120\end{array}$ & $\begin{array}{c}33,4 \\
68 \\
86 \\
96,5 \\
108\end{array}$ & $\begin{array}{c}33,3 \\
68 \\
87 \\
97 \\
107\end{array}$ & $\begin{array}{r}33,4 \\
68 \\
86,5 \\
96,7 \\
107,5\end{array}$ & $\begin{array}{l}6,6 \\
12 \\
13,5 \\
13,3 \\
12,5\end{array}$ & dto. \\
\hline
\end{tabular}


Tabelle 4.

\begin{tabular}{|c|c|c|c|c|c|c|}
\hline $\begin{array}{c}\text { Farbe } \\
\text { und } \\
\text { Helligkeit }\end{array}$ & $\begin{array}{l}\text { Oeffnung } \\
\text { d. grossen } \\
\text { Sectoren } \\
\text { von } 0^{\circ} \text { bis }\end{array}$ & $\begin{array}{r}\text { Stand } \\
\text { Secto } \\
\text { gefur } \\
\text { d.kleine } \\
\text { Zah }\end{array}$ & $\begin{array}{l}\text { kleinen } \\
\text { andes, } \\
\text { a von } \\
\text { grossen } \\
\text { her }\end{array}$ & Mittel & $\begin{array}{l}\text { Diffe- } \\
\text { renz }\end{array}$ & $\begin{array}{c}\text { Rotationsgeschwin- } \\
\text { digkeit }\end{array}$ \\
\hline Blau $1 / 2$ & $\begin{array}{r}40 \\
80 \\
100 \\
120 \\
140\end{array}$ & $\begin{array}{r}34 \\
70 \\
87 \\
108 \\
129 \\
\end{array}$ & $\begin{array}{c}33 ! ! \\
69 \\
87,5 \\
109 \\
128,5 \\
\end{array}$ & \begin{tabular}{|c|}
34 \\
69,5 \\
87,3 \\
108,5 \\
128.7 \\
\end{tabular} & $\begin{array}{c}6 \\
10,5 \\
12,7 \\
11,5 \\
11,3\end{array}$ & $\begin{array}{c}\text { Rotat. } 3,33 \text { in } 1 \text { Sec. } \\
1^{\circ}=1 / 1200 \mathrm{Sec} .\end{array}$ \\
\hline Blau $1 / 2$ & $\begin{array}{r}40 \\
80 \\
100 \\
120 \\
140 \\
\end{array}$ & $\begin{array}{c}36 \\
71,2 \\
89,5 \\
108,8 \\
129,5\end{array}$ & $\begin{array}{l}35 ! ! \\
72 \\
90,4 \\
109 \\
130\end{array}$ & $\begin{array}{c}35,6 \\
71,6 \\
90 \\
108,9 \\
129,8\end{array}$ & $\begin{array}{c}4,4 \\
8,4 \\
10 \\
11,1 \\
10,2\end{array}$ & dto. \\
\hline Blau 1 & $\begin{array}{r}30 \\
60 \\
80 \\
100 \\
120 \\
140\end{array}$ & $\begin{array}{c}23,7 \\
44 \\
60 \\
74 \\
94 \\
116\end{array}$ & $\begin{array}{c}22 ! \\
44,4 \\
61,5 \\
75 \\
96 \\
115\end{array}$ & $\begin{array}{c}22,8 \\
44,2 \\
60,8 \\
74,5 \\
95 \\
115,5\end{array}$ & $\begin{array}{r}7,2 \\
15,8 \\
19,2 \\
25,5 \\
25 \\
24,5\end{array}$ & dto. \\
\hline Blau 1 & $\begin{array}{r}40 \\
80 \\
100 \\
120 \\
140 \\
160\end{array}$ & $\begin{array}{c}29 \\
62 \\
77,8 \\
96 \\
117 \\
139\end{array}$ & $\begin{array}{c}29 \\
60,5 \\
78,2 \\
96 \\
117 \\
138,5\end{array}$ & $\begin{array}{c}29 \\
61,2 \\
78 \\
96 \\
117 \\
138,8\end{array}$ & $\begin{array}{l}11 \\
18,8 \\
22 \\
24 \\
23 \\
21,2\end{array}$ & dto. \\
\hline
\end{tabular}

Tabelle 5.

\begin{tabular}{|c|c|c|c|c|}
\hline Eingestellte Farbe & $\begin{array}{l}\text { Weite d. } \\
\text { Spaltes } \\
\text { in Mm. }\end{array}$ & $\begin{array}{c}\text { Oeffnung } \\
\text { der } \\
\text { Sectoren }\end{array}$ & 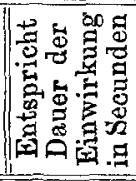 & Stattgehabte Empfindung \\
\hline Roth..... & $\begin{array}{l}0,098 \\
0,39\end{array}$ & $\begin{array}{l}1^{\circ} \\
2^{\circ}\end{array}$ & $\begin{array}{l}0,00029 \\
0,000578\end{array}$ & $\begin{array}{l}\text { Keine. } \\
\text { Rechts roth, linker Theil des } \\
\text { Gesichtsfeldes dunkel. }\end{array}$ \\
\hline Orange u. Gelb.. & & $\begin{array}{l}4^{\circ} \\
1^{\circ}\end{array}$ & & $\begin{array}{l}\text { Links roth, rechts gelb. } \\
\text { Links roth. rechts gelb. }\end{array}$ \\
\hline $\begin{array}{l}\text { Orange bis Grün- } \\
\text { lichgelb....... }\end{array}$ & $\begin{array}{l}0,098 \\
0,195 \\
0,39\end{array}$ & $\begin{array}{l}3^{\circ} \\
6^{\circ} \\
6^{\circ}\end{array}$ & $\begin{array}{l}0,000868 \\
0,001736 \\
0,001736\end{array}$ & $\begin{array}{l}\text { Rechts blau, links gelb. } \\
\text { Rechts blau, links gelb. } \\
\text { Rechts grün, links gelb mit } \\
\text { roth. }\end{array}$ \\
\hline $\begin{array}{l}\text { Grün - links } \\
\text { Grünlichgelb }\end{array}$ & $\begin{array}{l}0,585 \\
0,195 \\
0,098 \\
0,098 \\
0,098 \\
0,098\end{array}$ & $\begin{array}{ll}1 & 0 \\
1 & 0 \\
1 & 0 \\
3 & 0 \\
6^{\circ} \\
3^{\circ}\end{array}$ & $\begin{array}{l}0,00029 \\
0,00029 \\
0,00029 \\
0,000868 \\
0,001736 \\
0,000868\end{array}$ & $\begin{array}{l}\text { Grün, deutlich. } \\
\text { Blaugrün. } \\
\text { Deutlich blau. } \\
\text { Blau. } \\
\text { Grün. } \\
\text { Blau, das Grün ist weg. }\end{array}$ \\
\hline
\end{tabular}




\begin{tabular}{|c|c|c|c|c|}
\hline Eingestellte Farbe & $\begin{array}{l}\text { Weite d. } \\
\text { Spaltes } \\
\text { in } \mathrm{Mm} .\end{array}$ & $\begin{array}{c}\text { Oeffnung } \\
\text { der } \\
\text { Sectoren }\end{array}$ & 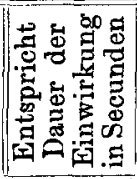 & Stattgehabte Empfindung \\
\hline \multirow[t]{8}{*}{ Grün......... } & 0,098 & $1^{0}$ & 0,00029 & Schwach blau. \\
\hline & 0,049 & $1^{0}$ & 0.00029 & Lichtschimmer, keine Farbe. \\
\hline & 0,049 & $6^{0}$ & 0,001736 & Blaugrau. \\
\hline & 0,049 & $15^{\circ}$ & 0,00434 & Deutlich blan. \\
\hline & 0,39 & $1^{0}$ & 0,00029 & Blaugrün. \\
\hline & 0,39 & $6^{0}$ & 0,001736 & Grün. \\
\hline & 0,195 & 10 & 0,00029 & Deutlich blau. \\
\hline & 0,78 & $1^{0}$ & 0,00029 & Grün. \\
\hline \multirow[t]{3}{*}{ Blau } & 0,39 & $6^{0}$ & 0,001736 & Schön blau. \\
\hline & 0,39 & $3^{0}$ & 0,000868 & Blau. \\
\hline & 0,39 & $1^{0}$ & 0,00029 & Lichtschimmer, keine Farbe. \\
\hline
\end{tabular}

\section{Tabelle 6.}

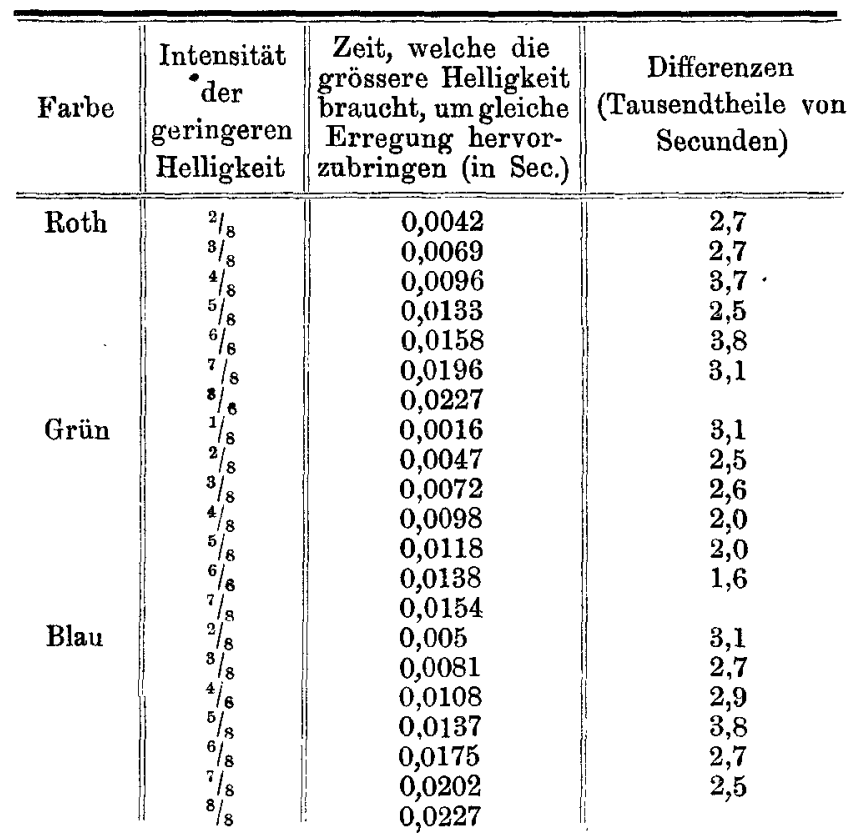

Ich habe schliesslich noch die ehrenvolle Pflicht zu erfüllen, Hrn. Professor Fick für die ausgedehnte Unterstützung, die er im Verlaufe dieser Untersuchung mir zu Theil werden liess, meinen besten Dank auszusprechen. 

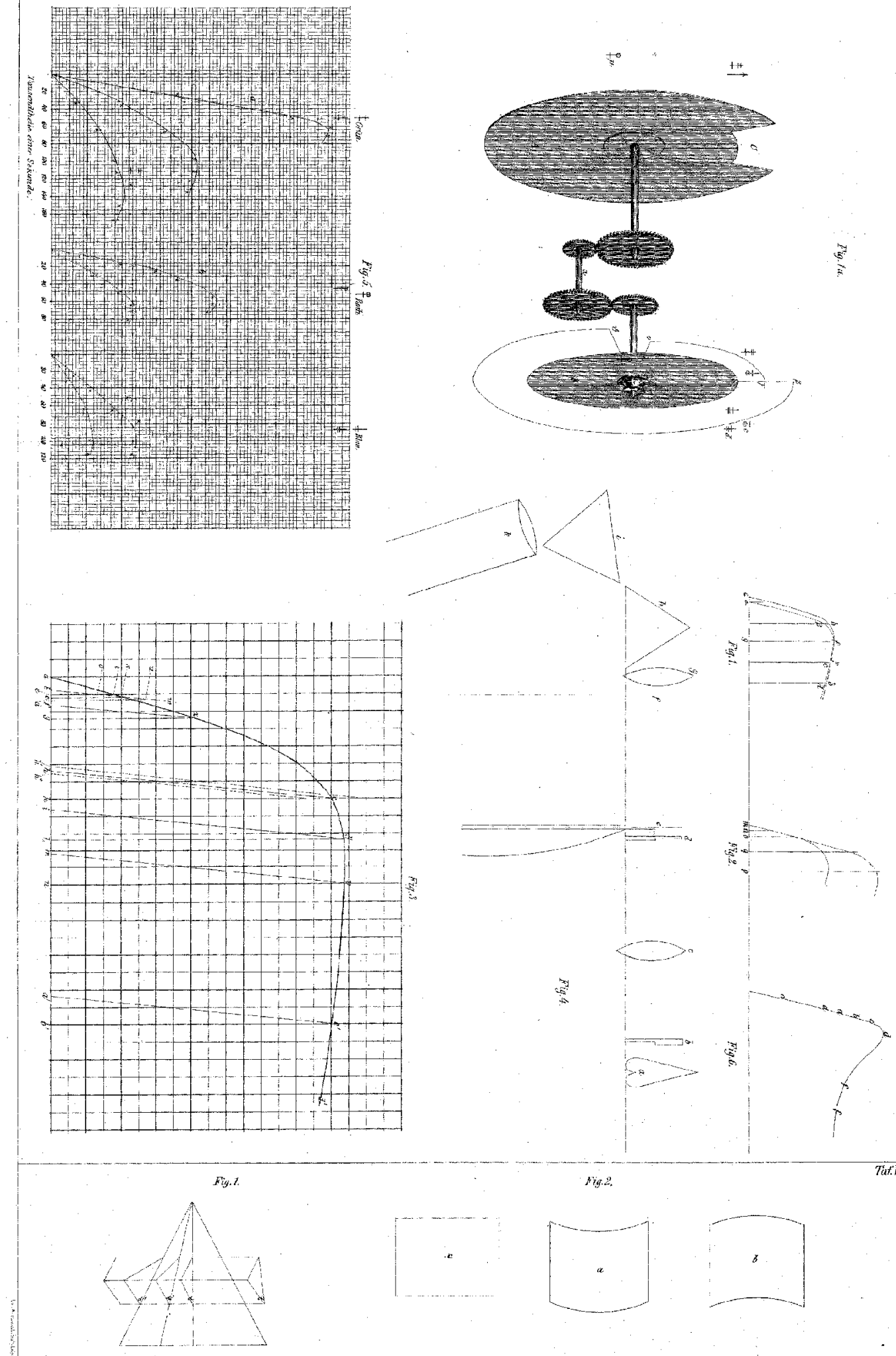

772.

Fig.t.
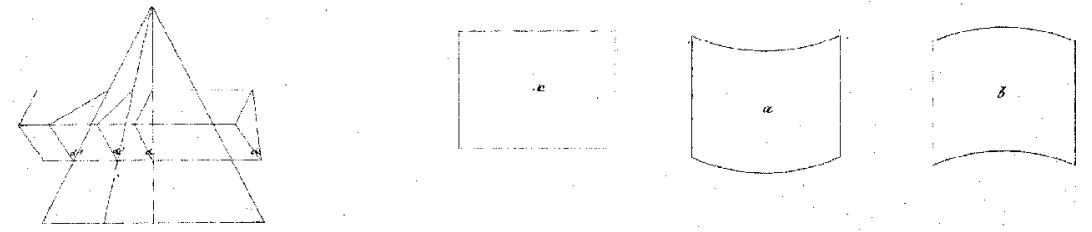